\title{
Lombalgia em eqüinos
}

\section{Ana Liz Garcia ALVES ${ }^{1}$}

Brunna Patricia Almeida da FONSECA ${ }^{1}$

Armen THOMASSIAN ${ }^{1}$

José Luiz de Mello

NICOLETTI ${ }^{1}$

Carlos Alberto HUSSNI ${ }^{1}$

Andressa Batista da

SILVEIRA ${ }^{\top}$

\section{Correspondência para:}

Brunna Patrícia Almeida da Fonseca

FMVZ - UNESP - Departamento de

Cirurgia e Anestesiologia Veterinária

Distrito de Rubião Júnior, s/n Caixa postal

560 - Botucatu/SP

E-mail: bfonseca@fmvz.unesp.br

Recebido para publicação: 20/06/2005 Aprovado para publicação: 07/02/2007

1 - Departamento de Cirurgia e Anestesiologia, Faculdade de Medicina

Veterinária e Zootecnia, Universidade Estadual Paulista, Botucatu - SP

\section{Resumo}

As lombalgias, sejam elas de origem primária ou secundária, são uma causa importante para a queda de desempenho atlético em eqüinos, mas o tamanho e a biomecânica complexa dificultam o diagnóstico e tratamento desta enfermidade. Sendo assim, o conhecimento da anatomia desta região é de grande importância para a semiologia toracolombar. O diagnóstico das lombalgias se faz por meio do exame físico e dos exames complementares, representados pelos métodos de diagnóstico por imagem, tais como a radiografia, a ultra-sonografia e a termografia. As principais afecções causadoras das lombalgias nos eqüinos são o contato entre processos espinhosos, a desmite supraespinhosa, a osteoartrite dos processos articulares e lesões dos corpos e discos vertebrais. Os principais tratamentos utilizados para estas lesões são os antiinflamatórios não esteroidais, infiltrações locais, acupuntura, fisioterapia, manejo do treinamento e cirurgia.

\section{Introdução}

O mercado eqüestre nacional movimenta anualmente cerca de 7,3 bilhões de reais, sendo que a quantidade movimentada pelo setor esportivo corresponde a aproximadamente 900 milhões de reais ${ }^{1}$. Nos eqüinos a principal causa de inatividade atlética são as claudicações, gerando perdas econômicas. As lombalgias representam 4,35\% da casuística de afecções locomotoras ${ }^{2}$, um número elevado, quando se analisa as várias enfermidades que acometem o sistema músculo-esquelético dos eqüinos.

A dor lombar é uma das queixas mais comuns de incapacidade física na medicina humana, resultando em dor crônica, restrição da movimentação, estresse e, perdas econômicas decorrentes dos gastos com cuidados médicos e diminuição da produtividade ${ }^{3}$. Apesar disso, as lombalgias ainda são pouco exploradas, em função de vários fatores, como a deficiência no conhecimento da anatomia funcional da coluna vertebral toracolombar, a falta de informações a respeito da etiopatogenia das enfermidades e a dificuldade de acesso às estruturas anatômicas envolvidas por meio da palpação e imagens diagnósticas ${ }^{4,5,6}$. O tamanho e a anatomia funcional complexa, em conjunto com a alta exigência da coluna vertebral toracolombar dos eqüinos na prática das modalidades atléticas, os predispõem a lesões que podem levar à dor ou disfunções locomotoras ${ }^{7}$.

\section{Anatomia}

A coluna toracolombar é composta por 18 vértebras torácicas (T), seis vértebras lombares $(\mathrm{L})$ e cinco vértebras sacrais $(\mathrm{S})$ fundidas. Alguns eqüinos possuem variações congênitas nas articulações cervicotorácicas, toracolombares e lombossacras, sendo a mais comum a fusão da ultima vértebra lombar com a primeira sacral, conhecida como sacralização da sexta vértebra lombar. A anquilose intervertebral altera a biomecânica da região das vértebras envolvidas, especialmente na junção lombossacra, pois esta é a articulação com maior mobilidade entre T2 e S1. A anquilose lombossacra aumenta o estresse sobre as articulações lombares caudais, e pode predispor o desenvolvimento de osteoartrite dos processos articulares e lesões dos discos intervertebrais. A fusão dos processos 
transversos também pode ocorrer e possui efeito similar sobre as articulações intervertebrais adjacentes, porem de menor intensidade, uma vez que estas articulações possuem uma menor amplitude de movimento ${ }^{8}$.

As primeiras 10 vértebras torácicas possuem longos processos espinhosos, que servem como ponto de inserção para o ligamento supraespinhoso que possuem uma orientação dorsocaudal até T16, e se apresenta perpendicular ao eixo vertebral, sendo denominada vértebra anticlinal. Caudal a esta vértebra, os processos espinhosos passam a possuir orientação dorsocranial. $\mathrm{Na}$ maioria dos cavalos os processos espinhosos de L6 e S1 são divergentes, permitindo um amplo movimento ventrodorsal da articulação lombossacra. Os processos transversos entre L5 e L6 e entre L6 e S1 (algumas vezes entre L4 e L5) se articulam por meio de diartroses, denominadas articulações intertransversas, que limitam a movimentação lateral desta área. Os corpos vertebrais lombares são maiores que das torácicas e possuem uma crista central que serve para inserção do diafragma $a^{8,9}$.

A estabilidade das vértebras da coluna toracolombar é promovida pelos ligamentos supra e interespinhoso, articulações dos processos articulares, articulações entre os corpos vertebrais e pelos ligamentos longitudinal ventral e dorsal ${ }^{10}$. Esses ligamentos são mais espessos e elásticos na região torácica cranial e média, permitindo maior movimento a esta região, quando comparada à região torácica caudal e lombar ${ }^{9,11}$.

Os processos articulares craniais e caudais se articulam por meio de uma diartrose, que se encontram na base dos processos espinhosos, localizadas simetricamente de cada lado do plano mediano. Até T12, possuem uma única e plana faceta articular e entre T12 e T16 são duas facetas articulares anguladas. De T17 até S1 tais facetas são congruentes, com formato cilíndrico alinhadas com o eixo paramediano ${ }^{12}$. Essas variações regionais estão relacionadas com a mobilidade reduzida da região lombar e da maior amplitude de movimento da região torácica ${ }^{8}$. Os corpos vertebrais são estabilizados por articulações compostas por um disco intervertebral fibroso e dois ligamentos longitudinais. O ligamento longitudinal ventral é substituído pelo músculo longo do pescoço na região torácica cranial. Já ligamento longitudinal dorsal se localiza no canal vertebral e se adere a borda dorsal do disco intervertebral ${ }^{8,12}$.

A coluna vertebral é movimentada por músculos potentes. Os músculos epaxiais, localizados dorsalmente ao eixo vertebral, são responsáveis pela dorsiflexão quando contraídos bilateralmente. A contração unilateral gera uma flexão lateral e contribui para a rotação da coluna. Estudos eletromiográficos mostram que os músculos epaxiais limitam a ventroflexão e estabilizam a coluna vertebral durante a fase de suspensão do trote ${ }^{12,13,14}$. Os músculos epaxiais incluem o músculo espinhoso, músculo longuíssimo dorsal, músculo iliocostal e músculo multífidus. O músculo longuíssimo dorsal é o músculo mais forte. O músculo iliocostal é menor, mas desempenha um papel importante na flexão lateral devido à sua localização excêntrica. O músculo multífidus se localiza sob o músculo espinhoso e está em contato direto com as vértebras. Este músculo desempenha grande papel na estabilidade e propriocepção vertebral ${ }^{4,8,10}$.

Os músculos hipaxiais, localizados ventralmente ao eixo vertebral, agem sobre a ventroflexão quando contraídos bilateralmente. A contração unilateral induz á flexão lateral e contribui para a rotação. Os músculos hipaxiais incluem o psoas menor e maior e reto oblíquo ${ }^{15}$. Os músculos psoas menor e maior se inserem na face ventral das vértebras lombares, sob os processos transversos, e três últimas torácicas. Eles agem principalmente na articulação lombossacra, mas também agem sobre a articulação toracolombar e coluna lombar ${ }^{13,14}$. 


\section{Exame Clínico}

Os objetivos do exame clínico da região toracolombar dos eqüinos são: determinar se há dor, sua localização e as possíveis lesões presentes ${ }^{10}$.

\section{Exame físico}

O exame físico é essencial para o diagnóstico das lombalgias, sendo de grande importância a avaliação do eqüino como um todo, para identificar problemas em potencial que possam estar contribuindo para a claudicação e/ou queda de performance ${ }^{8,15}$.

Inspeção: As curvaturas anormais mais comuns de serem encontradas nos eqüinos são a cifose lombar e a lordose torácica, sendo que essas duas alterações podem ser encontradas em conjunto. Atrofia dos músculos epaxiais na região lombossacra resulta na proeminência dos processos espinhosos e aparente cifose da coluna lombar. A atrofia dos músculos epaxiais é um dos sinais de um animal potencialmente portador de lesões toracolombares, pois a atrofia reflete a redução de movimento em áreas de dor ${ }^{8,16}$. A lordose torácica pode ser observada em animais clinicamente sadios e não implica necessariamente na presença de dor lombar ${ }^{8,17}$.

Áreas de edema na região de apoio da sela ou falhas de pêlo refletem um mau ajustamento da sela no animal ou um mau posicionamento do cavaleiro ${ }^{16}$, pois estes dois últimos causam lesões na região toracolombar por trauma direto e/ou por causar um desequilíbrio nos movimentos do animal.

Palpação: A palpação da região toracolombar deve ser realizada com o animal em apoio quadrupedal. Deve-se aguardar até que o animal se acostume com a presença do examinador, principalmente no caso de animais com comportamento agitado, para que as reações não sejam mal interpretadas ${ }^{15}$.

A palpação das estruturas superficiais da região toracolombar auxilia na identificação de aumentos de volume e má formação ou mau alinhamento dos processos espinhosos. Identificação e localização de pontos de tensão muscular podem ser a chave para a presença de lesões significantes ${ }^{4}$.

A pressão sobre as estruturas superficiais como o ligamento supraespinhoso e músculos epaxiais é útil para a localização de pontos de dor. O tamanho dos músculos epaxiais impede a palpação de estruturas profundas ${ }^{8}$.

Testes de mobilização: A estimulação de movimento da coluna vertebral toracolombar é importante para se avaliar a quantidade de movimento tolerada pelo eqüino e manifestação de dor durante o movimento, como tensão da musculatura toracolombar, movimentos de cauda e flexão dos membros. Recomendase o seguinte protocolo: (1) ventro e dorsoflexão torácica e lombar; (2) flexão lateral e rotação toracolombar e (3) flexão lateral e rotação cervical e torácica ${ }^{15}$.

Deve-se avaliar quais movimentos estão diminuídos ou não são tolerados para identificar possíveis locais de dor. Esses movimentos podem ser induzidos por estímulos cutâneos nas regiões dorsal e lateral do tronco e membros posteriores. Enquanto alguns animais respondem a estímulos digitais leves, outros necessitam de um estímulo mais intenso, que pode ser realizado com um instrumento de ponta romba, que é deslizado sobre o dorso do animal no sentido craniocaudal para a indução da ventro e dorsiflexão. Em animais de temperamento linfático pode ser necessária uma pressão maior para a indução desse movimento. Um eqüino hígido realiza estes movimentos em resposta ao estímulo de maneira suave e repetidas vezes ${ }^{8,15}$.

Exame durante o movimento: A avaliação do animal a passo, trote e galope é essencial para se identificar a presença de dor 
e alterações funcionais, tais como diminuição de mobilidade intervertebral em determinada área. O animal deve ser examinado em movimentos em linha reta e em círculos ao passo e trote em piso duro e ao trote e galope em piso macio para a identificação de alterações de mobilidade ${ }^{8}$.

\section{Exames complementares}

Bloqueios anestésicos regionais podem ser úteis na identificação do significado clínico de lesões superficiais, tais como desmites supraespinhosas e conflito dos processos espinhosos. O volume de anestésico utilizado é de 3 a $4 \mathrm{ml}$ por ponto (lidocaína $2 \%$ ), utilizando-se uma agulha de 4 a $5 \mathrm{~cm}$. As infiltrações são realizadas na região paravertebral, bilateralmente a cada $6 \mathrm{~cm}$, na área o áreas suspeitas de lesão, observando-se a resposta ao bloqueio após 15 a 20 minutos. A agulha entra paralela ao processo espinhoso, no músculo Longuíssimo dorsal.

O bloqueio de estruturas mais profundas, como dos processos articulares pode gerar interpretações confusas, pois o anestésico pode se difundir até atingir os ramos ventral e dorsal dos nervos espinhais. Os bloqueios anestésicos possuem pouco valor no diagnóstico de lesões nos discos intervertebrais e espondiloses ${ }^{8,15}$, pois não é possível a infiltração das estruturas hipaxiais, pois estas se encontram abaixo do gradil costal e processos transversos.

\section{Diagnóstico por imagem}

Devido à inacessibilidade de muitas estruturas da coluna vertebral que podem estar afetadas, técnicas diagnósticas auxiliares como a radiografia, ultra-sonografia e a termografia são particularmente importantes no exame de um eqüino com suspeita de alteração toracolombar ${ }^{6}$.

a) Termografia

A termografia tem sido utilizada em eqüinos como exame complementar no diagnóstico e prognóstico de lesões inflamatórias ${ }^{18,19}$, pois demonstra alterações na temperatura superficial cutânea. Nos casos de lombalgias, a termografia possui sua maior utilidade no mapeamento das possíveis lesões presentes na região toracolombar. Pode-se encontrar dois padrões de imagens térmicas distintas sugestivos de lesões: manchas quentes e manchas frias. As primeiras são causadas por aumento de temperatura local, causada por processos inflamatórios, como desmites supraespinhosa, por exemplo. Já as manchas frias são causadas por lesões como a osteoartrite intervertebral dorsal, que causam dor, estimulando os troncos nervosos simpáticos que emergem pelos forames intervertebrais e enervam tanto a articulação como a pele e musculatura. Como conseqüência deste estímulo, há uma vasoconstrição reflexa, que causa uma queda ta temperatura superficial local formando uma mancha fria no termograma. Como detecta o status fisiológico da região examinada, a termografia é útil na verificação do significado clinico de lesões encontradas no exame radiográfico e ultra-sonográfico ${ }^{17}$.

b) Radiografia

As indicações para realização do exame radiográfico da coluna toracolombar incluem queixas de lombalgias por parte de proprietários, queda de performance, suspeitas clínicas e investigações de claudicações obscuras ${ }^{20}$.

O animal deve estar em apoio quadrupedal. É necessário um aparelho de alta potência $(80 \mathrm{~kW})$. Os cassetes devem ser posicionados o mais próximo possível do animal. O aparelho deve ser posicionado perpendicular ao eixo vertebral. Necessitase de pelo menos cinco posições para uma avaliação completa da coluna toracolombar entre T10 e L4. Em alguns eqüinos conseguese observar $\mathrm{L} 5^{8,20}$.

Por meio do exame radiográfico, é possível a visualização de lesões nas estruturas ósseas ("kissing spines", osteoartrite intervertebral dorsal, fraturas de processos espinhosos e corpos vertebrais e espondiloses) ${ }^{20}$. 
c) Ultra-sonografia

E possível o exame ultra-sonográfico de todas as estruturas epaxiais. A imagem dos processos espinhosos e ligamentos associados pode ser realizada com transdutores de $7,5 \mathrm{MHz}$ ou $10 \mathrm{MHz}$. Uma almofada acústica é útil no exame das estruturas mais superficiais. O exame dos processos articular e transverso pode ser feitos com transdutores de 5,0 MHz até 2,5 $\mathrm{MHz}$. Imagens longitudinais medianas e paramedianas devem ser combinadas com imagens transversais para se examinar todas as estruturas vertebrais ${ }^{11,21}$.

A ultra-sonografia é principalmente utilizada no exame das partes moles componentes da região toracolombar, como o ligamento supra e interespinhoso e musculatura, mas também possui grande valor no diagnóstico da osteoartrite intervertebral dorsal, principalmente quando localizada caudal a L4, onde o exame radiográfico não é possível de ser realizado. Outro uso desta técnica diagnóstica é no exame da articulação lombossacra, pela via transretal ${ }^{11}$.

\section{Lesões}

Sobreposição dos processos espinhosos ou "kissing spines"

Esta é a enfermidade mais conhecida da região toracolombar do eqüino, sendo considerada uma síndrome, por envolver diversos tipos de lesões. A localização mais comum de ocorrência deste tipo de lesão é no segmento de coluna entre T10 e T18, no entanto, pode ocorrer entre L1 e L6. O remodelamento ou fraturas por avulsão da porção dorsal dos processos espinhosos reflete uma lesão de inserção do ligamento supraespinhoso.

A sobreposição dos processos espinhosos é uma condição onde ocorre uma orientação anormal dos processos espinhosos. O contorno na porção média dos processos espinhosos pode estar alterada devido a formação de entesófitos na inserção do ligamento interespinhoso ${ }^{22}$. $\mathrm{O}$ diagnóstico destas lesões é feito por meio do exame radiográfico, mas o exame ultrasonográfico é útil na averiguação da existência de desmites supra e interespinhosas concomitantes ${ }^{8,11}$.

"Kissing spines" pode ser encontrada em eqüinos atletas sem manifestações de lombalgia. Desta forma, o significado clínico desta alteração deve ser cuidadosamente analisado. Os achados radiográficos devem ser relacionados com o exame físico e à resposta aos bloqueios anestésicos ${ }^{8,22}$. a presença desta lesão deve ser investigada em animais que apresentam uma diminuição da mobilidade da região torácica e toracolombar, em animais que praticam modalidades esportivas que predispõem ao aparecimento desta lesão, como o salto e em animais da raça Puro Sangue Inglês, pois este apresentam processos espinhosos mais longos ${ }^{22}$.

\section{Desmite supraespinhosa}

Lesões do ligamento supraespinhoso ocorrem geralmente entre T15 e L3 e podem ser associadas com aumento de volume local e dor à palpação. Estas lesões ocorrem geralmente sobre os processos espinhosos, podendo se estender entre dois processos adjacentes e são facilmente diagnosticadas por meio da ultra-sonografia. O aumento de volume local do ligamento produz uma deformação dorsal da região toracolombar, a qual pode ser examinada pela ultrasonografia ${ }^{8}$. Lesões hipoecóicas no ligamento são compatíveis com desmite aguda ou crônica; já focos hiperecóicos, com ou sem sombras acústicas podem ser vistos no caso de desmopatia crônica. Desmopatias de inserção (entesopatias) podem ser identificadas por irregularidades na superfície dorsal dos processos espinhosos. Devendose observar a presença de áreas com alterações de ecogenicidade e de orientação de fibras ${ }^{11,21}$.

Lesões no ligamento supraespinhoso causam dor no animal no momento da ventroflexão, onde há um aumento da tensão sobre esta estrutura, sendo prejudicial em animais que praticam modalidades 
esportivas que exigem uma posição de ventroflexão constante, como nas provas de apartação.

\section{Osteoartrite intervertebral}

As articulações intervertebrais sinoviais formadas pelos processos articulares estão localizadas dorsalmente ao canal vertebral e são compostas pelo processo articular caudal de uma vértebra, espaço articular e pelo processo articular cranial da vértebra imediatamente caudal ${ }^{22}$.

Oito tipos de achados radiográficos podem ser associados à osteoartrite dos processos articulares nos eqüinos: (1) Assimetria, (2) modificação da radiopacidade do processo articular, (3) áreas radioluscentes no osso subcondral, (4) proliferação peri-articular, (5) proliferação ventral, (6) anquilose, (7) osteólise das articulações intervertebrais e (8) fratura. Estes achados são encontrados na maioria das vezes na articulação toracolombar e na região lombar. Os tipos 2, 4 e 6 são mais comuns entre T16 e L3. Já os tipos 5 e 7 são mais comuns na região lombar. Proliferações periarticulares dorsais (tipos 4 e 6 ) podem ser identificados pela ultra-sonografia, determinando se a lesão é simétrica ou, caso não seja, qual antímero esta mais afetado. Este tipo de lesão é mais passível de causar lombalgias em eqüinos do que o "kissing spines" ". As manifestações clínicas mais comuns desta lesão são: dor na musculatura epaxial, principalmente lombar; diminuição da amplitude de movimento da articulação lombossacra; tensão da musculatura abdominal e diminuição da amplitude da passada dos membros posteriores (galope de lebre).

\section{Lesão de corpos e discos vertebrais}

Lesões de corpo vertebral são pouco comuns em eqüinos e não podem ser observadas por meio da ultra-sonografia na região toracolombar, a não ser caudalmente a L4, por meio do acesso transretal ${ }^{11,12}$, sendo a radiografia a técnica diagnóstica de escolha.
Dentre as lesões se incluem: (1) proliferações ventrais, ventrolaterais ou laterais (espondilose vertebral); (2) deformações do corpo vertebral; (3) deformação da cabeça e fossa da vértebra adjacente (entesopatia de disco) ${ }^{8}$.

A presença de espondiloses em uma ou mais vértebras pode acarretar a compressão de ramos nervosos pela diminuição no diâmetro dos forames intervertebrais. Anquiloses podem predispor a ocorrência de fraturas, pela redução da capacidade vertebral de absorção e transferência das forças de locomoção. Fraturas e luxações das vértebras lombares geralmente resultam em sinais neurológicos, mas ocasionalmente podem ocorrer sinais intensos de dor aguda, com rápida atrofia da musculatura epaxial ${ }^{22}$.

\section{Prognóstico}

O prognóstico de eqüinos com qualquer lesão na região toracolombar depende de diversos fatores, incluindo tolerância individual à dor, habilidade do treinador, tipo de trabalho e tipo, número e severidade das lesões. Eqüinos que apresentam desmite aguda supraespinhosa possuem um prognóstico bom, enquanto animais com lesões dos processos articulares possuem prognóstico ruim. Animais que apresentam conflito dos processos espinhosos possuem um prognóstico reservado. O prognóstico nos casos de espondilose vertebral varia muito, de acordo com a tolerância do paciente, mas geralmente se observa que quanto maior o numero de vértebras acometidas, pior o prognóstico ${ }^{8,15,22}$.

Jeffcott ${ }^{23}$ realizou um estudo com 190 animais com lombalgias crônicas relacionando o tipo de lesão ao retorno à atividade atlética, obtendo os seguintes resultados: lesões musculares e ligamentosas $79 \%$, espondiloses $9 \%$, kissing spines $57 \%$, lesões sacroiliacas $45 \%$.

\section{Tratamento}

O objetivo geral do tratamento de lombalgias é: eliminar a dor do animal o mais 
rápido possível, para permitir que o animal possa ser exercitado, evitando assim a perda de massa muscular e de condicionamento físico ${ }^{8}$.

\section{Tratamento sistêmico}

O tratamento de lombalgias com antiinflamatórios não esteroidais (AINEs) é geralmente ineficaz. Muitos animais são submetidos a exames da região toracolombar após a falta de resposta ao tratamento com AINEs; tratamento dos espasmos musculares com miorrelaxantes, como o tiocolchicoside é indicado em animais que apresentem espasmo da musculatura toracolombar ${ }^{8,24}$.

\section{Infiltrações locais}

Injeções periespinhais ou interespinhais de corticosteróides, associadas ou não a miorrelaxantes ou neurolíticos são utilizadas no tratamento de "kissing spines" e desmites inter e supraespinhosas. Nesses casos utilizase uma agulha de 4 a $5 \mathrm{~cm}$ de comprimento, para se injetar o líquido no músculo longuíssimo dorsal ou no espaço interespinhoso. Já o tratamento das osteoartrites do processo articular é feito com injeções paramedianas no músculo multífidus de ambos os lados na altura da lesão, a $2 \mathrm{~cm}$ do plano mediano, com agulhas de 9 a $11 \mathrm{~cm}$. Este tipo de infiltração é melhor realizado quando guiado por meio da ultrasonografia. Nesta situação o uso de neurolíticos é contra-indicado, devido à proximidade dos ramos dos nervos espinhais ${ }^{8,25}$.

\section{Acupuntura}

A acupuntura vem sendo relatada como uma prática efetiva no tratamento de lombalgias crônicas em humanos e animais, incluindo eqüinos, podendo ser utilizada como único tratamento, ou como adjuvante a outras terapias.

Em contraste com a lombalgia aguda a qual possui um caráter de dor nociceptivo, as lombalgias crônicas são muitas vezes caracterizadas pela ausência de lesões detectáveis, pois a dor crônica é originada pela irritação constante dos neurônios préganglionares aferentes, como nos casos de fibromialgias em humanos.

Existem algumas modalidades de acupuntura que podem ser utilizadas no tratamento das lombalgias, como a utilização de agulhas, a eletroacupuntura, onde se utiliza uma corrente elétrica para a estimulação dos acupontos e a aquapuntura, onde se injetam líquidos nos acupontos com o objetivo de manter o estimulo por um período prolongado $^{26}$.

\section{Fisioterapia}

A fisioterapia age nos tecidos responsáveis pela movimentação do sistema musculoesquelético: articulações, ligamentos, músculos e nervos, devendo ser considerada como uma extensão da terapia, auxiliando os animais no seu retorno às atividades físicas e atléticas ${ }^{4,27}$. Dentre as técnicas utilizadas na fisioterapia do sistema musculoesquelético do eqüino, podemos citar: (1) massagem, que possui como principais indicações o aumento de circulação sangüínea, redução da dor e redução de edemas nos locais lesionados, (2) terapia por campo magnético, indicado nos casos de inflamação e pós cirúrgico de fraturas; (3) estimulação muscular, indicada nos casos de atrofia muscular; (4) ultra-som terapêutico, indicado nos casos de hematomas, feridas, fibroses e para alívio da dor e (5) laser de baixa intensidade, indicado para os casos de lesões articulares, como capsulites e perda de sensibilidade cutânea ${ }^{4,28}$.

\section{Manejo do treinamento}

Com o animal medicado para redução da dor, a modificação do programa de treinamento é uma parte essencial do manejo das lombalgias. Os objetivos desta modificação são evitar a atrofia muscular e desenvolver o controle proprioceptivo toracolombar e estabilidade vertebral. Descanso é contraindicado, a não ser em animais irresponsivos 
ao tratamento ${ }^{8,12}$. Dentre as recomendações gerais se incluem:

- Conferir a sela, pois selas mal ajustadas causam dor e desconforto ao animal, prejudicando sua performance e podendo causar lesões toracolombares.

- Treinamento progressivo em animais jovens, pois os animais se encontram maduros fisicamente e mentalmente somente aos 4 a 5 anos. Sendo assim, animais jovens podem se lesionar mais facilmente com uma rotina de treinamento similar a de um animal adulto.

- Aquecimento progressivo, iniciando em piso macio sem sela e depois com a sela, para aquecer devidamente a musculatura de todo aparelho locomotor.

- Após o aquecimento, trabalhar em cânter antes do trote, pois no cânter a coluna realiza apenas um movimento de dorsoventroflexão ativo por passada, enquanto no trote a coluna realiza esse movimento duas vezes, de forma passiva, o que desfavorece o aquecimento da musculatura toracolombar.

- Identificar e remover exercícios que causem desconforto ao animal ${ }^{21}$. Estes podem ser identificados pela presença de espasticidade muscular anormal durante sua realização.

Alguns exercícios podem ser incluídos na rotina de aquecimento do animal, tais como a flexão cervical, pois esta induz a flexão torácica associada, forçando a separação dos processos espinhosos ${ }^{4,27}$.

\section{Tratamento cirúrgico}

O tratamento cirúrgico dos casos de "kissing spines" pela ressecção das tuberosidades dos processos espinhosos foi realizado com sucesso em animais irresponsivos ao tratamento conservativo dessa lesão. É importante a seleção de animais com lesões que envolvem apenas a porção dorsal dos processos espinhosos. É necessário um período de convalescença de 6 meses de maneira geral ${ }^{8}$. Lauk e Kreling ${ }^{25}$ realizaram este procedimento em 50 animais de salto, com um resultado de $54 \%$ de retorno à atividade atlética plena (27 animais). Walmsley et al. ${ }^{29}$ realizaram este procedimento em 288 animais de raças variadas em um período de 8 anos, com resultado de $72 \%$ de retorno à atividade atlética plena.

\section{Back pain in horses}

\section{Abstract}

Back pain, whether they have a primary or secondary origin, is a important cause to the drop of the equine performance, but the size and the complex biomechanics make it difficult to diagnosis and treat the injury. As it is, the knowledge of this anatomic site is of great importance to the thoracolumbar examination. The diagnosis of back pain is done through physical examination and complementary exams, represented by the image methods of diagnosis, such as radiography, ultrasonography and thermography. The most important lesions that cause the back pain in an equine are impingement of the dorsal spinous processes, desmopathy of the supraespinous ligament, osteoarthritis of the articular processes and lesions of the vertebral discs and body. The most common treatments to back pain are the non-steroidal anti-inflammatory drugs, local injections, acupuncture, physiotherapy, training management and surgery.
Key words: Thoracolumbar. Ultrasonography. Performance. Lameness. Vertebrae. Back pain. 


\section{Referências}

1 LIMA, R. A. S.; SHIROTA, R.; BARROS, G. S. C. Estudo do complexo do agronegócio cavalo. Piracicaba: Centro de Estudos Avançados em Economia AplicadaEsalq/USP, 2006. Disponível em: http:// www.cna.org.br. Acesso em: 22 ago 2006.

2 ALVES, A. L. G. et al. Estudo retrospectivo de dor lombar em eqüinos. Bras. J. Vet. Res. Animal Science, v. 41, p. 268-269, 2004. Supplement.

3 LECLAIRE, R. et al. Diagnostic accuracy of technologies used in low back pain assessment. Spine, v. 21, p. 1325-1331, 1996.

4 DENOIX, J. D.; PAILLOUX, J. P. Physical therapy and massage for the horse. 2. ed. Vermont: Trafalgar Square, 2005. 224 p.

5 JEFFCOTT, L. B. Historical perspective and clinical indication. Veterinary Clinics of North America Equine Practice, v. 15, p. 1-12, 1999.

6 VON SCHWEINITZ, D. G. Thermografic diagnostics in equine back pain. Veterinary Clinics of North America Equine Practice, v. 15, p. 161-178, 1999.

7 JEFFCOTT, L. B. Conditions causing thoracolumbar pain and dysfunction in horses. In: ANNUAL CONVENTION OF THE AMERICAN ASSOCIATION OF EQUINE PRACTIONERS, 31, 1985, Ontario,Toronto. Proceedings... Ontario:[s.n.], 1985. p. $285-296$.

8 DENOIX, J. D.; DYSON, S. J. Thoracolumbar Spine. In: ROSS, M. W.; DYSON, S. J. Diagnosis and management of lameness in the horse. 1. ed. Philadelphia: Saunders, 2003. p. 509-521.

9 DENOIX, J. D. Spinal biomecanics and functional anatomy. Veterinary Clinics of North America Equine Practice, v. 15, p. 27-60, 1999a.

10 SISSON, S. Músculos do eqüino. In: GETTY, R. Anatomia dos animais domésticos. 5. ed. Rio de Janeiro: Guanabara Koogan, 1986b. v. 1, p. 350-423.

11 DENOIX, J. D. Ultrasonographic evaluation of back lesions. Veterinary Clinics of North America Equine Practice, v. 15, p. 131-160, 1999b.

12 SISSON, S. Articulações dos equinos. In: GETTY, R. Anatomia dos animais domésticos. 5. ed. Rio de Janeiro: Guanabara Koogan, 1986a. v. 1, p. 324-349.

13 DENOIX, J. D.; AUDIGIÉ, F. The back and the neck. In: BACK, W.; CLAYTON, H. Equine locomotion. Philadelphia: Saunders, 2001. p.167-192.

14 ROBERT, C.; AUDIGIÉ, F.; VALETTE, J. P. Effects of treadmill speed on the mechanics of the back in the trotting saddle horse. Equine Veterinary Journal, v. 33, p. 154-159, 2001. Supplement.
15 STASHAK, T. S. Examination for lameness. In: STASHAK, T. S. Adam's lameness in horse. Baltmore: Williams \& Wilkins, 2002. p. 113-183.

16 MARTIN JR, B. B.; KLIDE, A. M. Physical examination of horses with back pain. Veterinary Clinics of North America Equine Practice, v. 15, p. 61-70, 1999.

17 TURNER, T. A. Diagnostic thermography. Veterinary Clinics of North America Equine Practice, v. 17, p. 95113, 2001.

18 EDDY, A. L.; VAN HOOGMOED, L. M.; SNYDER, J. R. The role of thermography in the manegement of equine lameness. Veterinary Journal, v. 162, p. 172181, 2001.

19 HEAD, M. J., DYSON, S. Talking the temperature of equine thermography. Veterinary Journal, v. 162, p. 166-167, 2001

20 BUTLER, J. A. et al. The Spine. In: Clinical radiology of the horse. Malden: Blackwell Science, 2000. p. 403453.

21 REEF, V. B. Musculoeskeletal Ultrasonography. In: REEF, V. B. Equine diagnostic ultrasound. Philadelphia: Saunders, 1998. p. 39-186.

22 HAUSSLER, K. K.; STOVER, S. M.; WILLITS, N. H. Pathologic changes in the lumbosacral vertebrae and pelvis in Thoroughbred horses. American Journal of Veterinay Research, v. 60, n. 2, p. 143-153, 1999.

23 HAUSSLER, K. K. The lower back and pelvis of performance horses receive a closer look. Journal of Equine Veterinary Science, v. 16, p. 279-281, 1996.

24 MARKS, D. Medical management of back pain. Veterinary Clinics of North America Equine Practice, v. 15, p. 179-194, 1999.

25 LAUK, H.; KRELING, I. Surgical treatment of kissing spines syndrome - 50 cases. II. Results. Pferdheilkunde, n.14, p. 123-130, 1998.

26 RIDGWAY, K. Acupuncture as a treatment modality for back problems. Veterinary Clinics of North America Equine Practice, v. 15, p. 211-221, 1999.

27 BROMILEY, M. W. Physical therapy in equine veterinary medicine: useful or useless? In: ANNUAL CONVENTION OF THE AMERICAN ASSOCIATION OF EQUINE PRACTIONERS, 46, 2000, Ontario,Toronto. Proceedings... Ontario:[s.n.], 2000. p. 94-97.

28 RIDGWAY, K.; HARMAN, J. Equine back rehabilitation. Veterinary Clinics of North America Equine Practice, v. 15, p. 263-280, 1999.

29 WALMSLEY, J. P. et al. Impingement of the dorsal spinous processes in two hundred and fifteen horses: case selection, surgical technique and results. Equine Veterinary Journal, v.34, p. 23-28, 2002. 